\section{Facharztprüfung zur Erlangung des Facharzttitels FMH für Dermatologie und Venerologie}

Aufgrund des Weiterbildungsprogrammes, welches am 1. Januar 2000 in Kraft gesetzt wurde, ist die Teilnahme an der Facharztprüfung für diejenigen Kandidatinnen und Kandidaten Voraussetzung für die Erlangung des Facharzttitels FMH für Dermatologie und Venerologie, welche ihre Weiterbildung in Dermatologie und Venerologie bis am 31. Dezember 2000 nicht abgeschlossen haben. Es empfiehlt sich, die Facharztprüfung frühestens im letzten Jahr der reglementarischen Weiterbildung abzulegen (Art. 23 WBO).
Ort: Dermatologische Universitätsklinik Zürich, Gloriastrasse 31, 8091 Zürich

Zeitpunkt: Donnerstag, 9. November 2000

Prüfungsgebühr: Die SGDV erhebt eine Prüfungsgebühr von Fr. 600.-.

Anmeldung: Die Anmeldeformulare können bezogen werden bei: Sekretariat Prof. S. Büchner, Dermatologische Universitätsklinik, Kantonsspital, Petersgraben 4, 4031 Basel, Tel. 06126550 99, Fax 0612654200.

Anmeldefrist: 15. Juli 2000

\section{Examen de spécialiste en vue de l'obtention du titre FMH en dermatologie et vénéréologie}

Conformément au programme de formation postgraduée entré en vigueur le $1^{\mathrm{er}}$ janvier 2000 , la participation à l'examen de spécialiste est une condition requise pour les candidats à l'obtention du titre de spécialiste FMH en dermatologie et vénéréologie qui n'ont pas terminé leur formation postgraduée en dermatologie et vénéréologie le 31 décembre 2000. Il leur est recommandé de passer l'examen de spécialiste au plus tôt durant la dernière année de sa formation postgraduée réglementaire (art. 23 RFP).
Lieu: Dermatologische Universitätsklinik Zürich, Gloriastrasse 31, 8091 Zürich

Date: Jeudi, 9 novembre 2000

Taxe d'examen: La SSDV prélève une taxe d'examen de Fr. 600.-.

Inscription: Les formulaires d'inscription peuvent être obtenus auprès du Secrétariat du Prof. S. Büchner, Clinique universitaire de dermatologie, Hôpital Cantonal, Petersgraben 4, 4031 Bâle, tél. 06126550 99, fax 0612654200.

Délai d'inscription: 15 juillet 2000 